\begin{tabular}{|c|l|}
\hline Title & $\begin{array}{l}\text { A facile method for synthesizing free shaped and tough double network hydrogels using physically crosslinked } \\
\text { poly(vinyl alcohol) as an internal mold }\end{array}$ \\
\hline Author(s) & Nakajima, Tasuku; Takedomi, Naoyuki; Kurokawa, Takay uki; Gong, Jian Ping \\
\hline Citation & $\begin{array}{l}\text { Polymer Chemistry, 1(5),693-697 } \\
\text { https://doi.org/10.1039/cOpy00031k }\end{array}$ \\
\hline Issue Date & 2010 \\
\hline Doc URL & http:/hdl.handle.net/2115/56612 \\
\hline Type & article (author version) \\
\hline File Information & PVADN_manuscript_accepted-3.pdf \\
\hline
\end{tabular}

Instructions for use 


\title{
A Facile Method for Synthesizing Free-Shaped and Tough Double Network Hydrogels via Physically Crosslinked Poly (Vinyl alcohol) as Internal Mold
}

\author{
Tasuku Nakajima, Naoyuki Takedomi, Takayuki Kurokawa, Hidemitsu Furukawa and Jian Ping Gong* \\ s Received (in $X X X, X X X)$ Xth $X X X X X X X X X 200 X$, Accepted Xth $X X X X X X X X X 200 X$ \\ First published on the web $X$ th $X X X X X X X X X 200 X$ \\ DOI: 10.1039/b000000x
}

The creation of Double Network hydrogels (DN gels), which show the extremely high mechanical strength, enabled hydrogels to apply both in medical and industrial fields. However, one obstacle 10 against various applications is the lack of formability of DN gels, owing to the brittleness of the first network PAMPS gels. In order to overcome this problem, we synthesized free-shaped DN gels (called PVA-DN gels) by using a physically cross-linked PVA gel as "internal mold". PVA-DN gels can form any complex shapes and their mechanical properties were comparable to those of conventional DN gels. This study may expand the application of tough hydrogels.

\section{${ }_{15}$ Introduction}

Polymer hydrogels, consisting of hydrophilic polymer network with large amount of water, are unique materials. They show many interesting functions, such as volume phase transition $^{1}$, low surface friction ${ }^{2}$, and permeability, which 20 cannot be implemented in solid materials. Thus, hydrogels have attracted much attention as novel functional materials from both medical and industrial fields. Although the strength of conventional hydrogels is typically too low to apply as any load-bearing materials, recent advance of synthesis enables to 25 improve the strength of hydrogels, such as Slide-ring gels, Nanocomposite gels, Double Network gels, Click gels, and Tetra-PEG gels ${ }^{3-7}$. Among them, the Double Network gels (DN gels), which were created by our group, show the highest fracture stress and toughness. DN gels are interpenetrating 30 network (IPN) hydrogels consisting of tightly cross-linked rigid poly(2-acrylamido-2-methylpropanesulfonic acid) (PAMPS) gels as the first network and loosely cross-linked flexible polyacrylamide (PAAm) as the second network (even if the cross-linker is not used, PAAm in common DN gels is 35 actually cross-linked ${ }^{8}$ ).

Although the DN gels contain $90 \mathrm{wt} \%$ of water (and only $10 \mathrm{wt} \%$ of polymer chains), they show the excellent mechanical properties. The compressive fracture stress of the DN gels reaches to several dozen $\mathrm{MPa}^{5,9}$, which is comparable 40 to that of articular cartilage $(36 \mathrm{MPa})^{10}$, a common natural tough hydrogel. In addition, their fracture energy is up to $2200 \mathrm{~J} / \mathrm{m}^{2} 8,11-13$, which is approximately 200 times larger than the theoretical value ${ }^{11}$. Some DN gels also exhibit suitability for regenerative medicine. Prof. Yasuda's group (Hokkaido ${ }_{45}$ University) has reported that the modified DN gels are excellently biocompatible and do not degrade in a body ${ }^{14,15}$. Additionally, they first succeeded in regenerating articular cartilage in vivo by embedding the biocompatible DN gels ${ }^{16}$. Based on these excellent mechanical and medical properties, ${ }_{50} \mathrm{DN}$ gels are supposed to become superior alternative materials for tough living organs, such as articular cartilages, blood vessels, and tendons.

Nevertheless, DN gels still have a problem - lack of formability, which is crucial for being extensively used as a ${ }_{55}$ material. For example, it is required for an artificial cartilage to form any specific complex shape dependent on the body of each patient. However, conventional DN gels can be formed only in the limited shapes, such as a sheet or a disc. The shapes of DN gels are determined by the first network ${ }_{60}$ PAMPS gels as "skeleton" due to the two-step polymerization. Whereas PAMPS gels themselves can be formed any shapes by using molds, it is very difficult to eject convoluted PAMPS gels without crashing due to their extremely poor mechanical properties, such as $0.08 \mathrm{MPa}$ of tensile fracture stress, 0.36 of ${ }_{65}$ tensile fracture strain and $0.8 \mathrm{~J} / \mathrm{m}^{2}$ of fracture energy ${ }^{11}$. Incidentally, PAMPS gels or DN gels also cannot be cut or scraped into desired shape after the synthesis.

We have solved this problem by applying "Double Network" structure. As above-mentioned, shape of DN gels is 70 determined by the first network. Based on this fact, it is imagined that if PAMPS gels are synthesized in another kind of hydrogels which are strong enough to eject from molds, the shape of them can be modified easily. We call these shapedeciding hydrogels "internal molds", as the opposite concept 75 of common "external" molds. This time, we chose physically cross-linked poly(vinyl alcohol) gels (PVA gels) as internal molds because they are so flexible and relatively strong that they can form any complicated shapes ${ }^{17-19}$. We performed the following experiments. Firstly, the PVA gels with complex 80 shapes were synthesized. Secondly, the PAMPS network was polymerized in the PVA gels. Thirdly, the PAAm network was synthesized in the presence of the PVA/PAMPS doublenetwork gels (PVA-PAMPS gels) and finally the free-shaped PVA/PAMPS/PAAm triple-network gels (PVA-DN gels) were 85 obtained. These PVA-DN gels show the similar mechanical properties to the conventional DN gels.

\section{Experimental}

\section{Materials}


Poly(vinyl alcohol) (PVA), polymerization degree: 2000 (Nakalai Tesque, Ltd.) was used as received. Dimethyl sulfoxide (DMSO, Junsei Chemical Co., Ltd.) was used as received. 2-Acrylamido-2-methylpropanesulfonic acid (AMPS, 5 Toa Gosei Co., Ltd.) was recrystallized from methanol. $N, N^{\prime}$ methylenebis(acrylamide) (MBAA, Wako Pure Chemical Industries, Ltd.) was recrystallized from ethanol. 2oxoglutaric acid (Wako Pure Chemical Industries, Ltd.) was used as received. Acrylamide (AAm, Junsei Chemical Co., ${ }_{10}$ Ltd.) was recrystallized from chloroform.

\section{Synthesis of PVA gels as internal mold}

The physically cross-linked PVA gels were prepared by quenching method ${ }^{18}$. Firstly, the 10wt\% PVA (DMSO: $\mathrm{H}_{2} \mathrm{O}=$ $3: 1$, w/w) solution was prepared by heating a mixture of the $15 \mathrm{PVA}$ and the solvent for $1 \mathrm{~h}$ at $90^{\circ} \mathrm{C}$. Then, the PVA solution was poured into various shapes of molds made of silicone rubber or glass, and they were quenched for $24 \mathrm{~h}$ at $-40^{\circ} \mathrm{C}$. After forming network structure, the gels were immersed in pure water more than 1 week to remove DMSO.

${ }_{20}$ Synthesis of free-shaped Double Network gels

First, the AMPS aqueous solution was prepared from $1 \mathrm{M}$ of AMPS as monomer, $4 \mathrm{~mol} \%$ of MBAA as cross-linker, and 0.1 or $0.6 \mathrm{~mol} \%$ of 2 -oxoglutaric acid as photoinitiator (the molar percentages are respective to the monomer). Then, the PVA 25 gels with various shapes were immersed in this solution for at least 3 days. After immersing, the PVA gels containing AMPS were let in an argon blanket and sealed to prevent drying; then PAMPS network was synthesized in the presence of PVA gels by UV polymerization for $8 \mathrm{~h}$. These PVA/PAMPS double30 network gels (called PVA-PAMPS gels) were then immersed in $2 \mathrm{M}$ of AAm and $0.01 \mathrm{~mol} \%$ of 2 -oxoglutaric acid aqueous solution for at least 2 days. Then, PAAm was synthesized in the presence of PVA-PAMPS gels by the same method. After the synthesis, PVA/PAMPS/PAAm triple-network gels, called 35 PVA-DN gels, were immersed in pure water more than 1 week in order to remove any un-reacted reagents. The swollen PVA-DN gels contained $90-92 \mathrm{wt} \%$ of water. It is important to synthesis the PVA-DN gels under no oxygen atmosphere. If the synthesis proceeds with oxygen, the surface of the sample 40 does not completely form gel state due to chain growth inhibition by oxygen ${ }^{20}$.

We also synthesized the PAMPS/PAAm double-network gels (conventional DN gels), the PVA/PAMPS doublenetwork gels, and the PVA/PAAm double-network gels as the 45 positive/negative controls with the same composition and by the same method, except that the second network of the PVA/PAAm gel was loosely cross-linked $(0.02 \mathrm{~mol} \%$ of MBAA was used). Table 1 shows the components of all the samples.

50

\section{Mechanical Strength Measurements}

The tensile-compressive tester (Tensilon RTC-1310A Orientic. Co.) was used for the measurement of mechanical properties. The compressive fracture stress $\sigma$ and the Young's 55 modulus $E$ were measured by compressive test on cylindrical

\begin{tabular}{cccc}
\hline Sample Name & Internal Mold & $1^{\text {st }}$ network & $2^{\text {nd }}$ network \\
\hline PVA gel & - & PVA & - \\
PAMPS gel & - & PAMPS & - \\
PAAm gel & - & PAAm & - \\
PVA-PAMPS gel & PVA & PAMPS & - \\
PVA-PAAm gel & PVA & PAAm & - \\
DN gel & - & PAMPS & PAAm \\
PVA-DN gel & PVA & PAMPS & PAAm \\
\hline
\end{tabular}

Table 1 The components of the samples used in this paper.
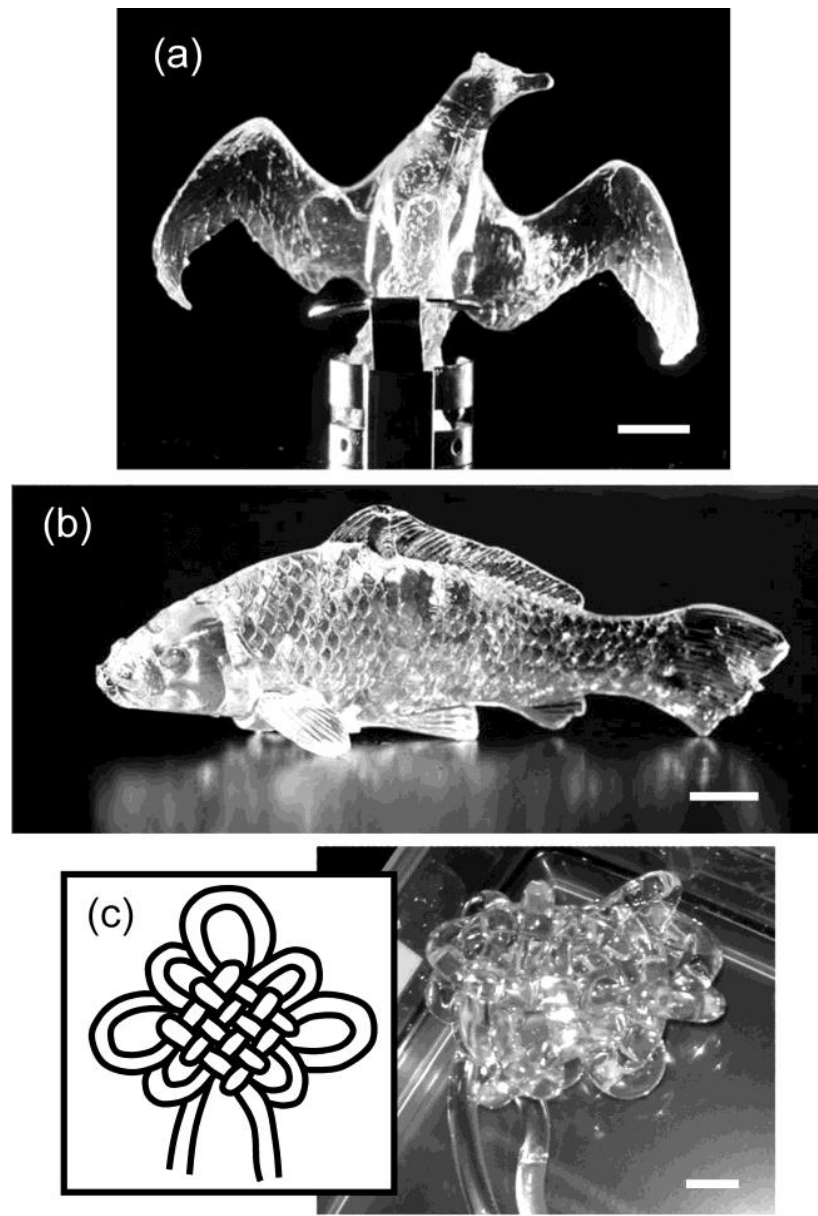

Fig. 1 The pictures of the PVA-DN gels with the shape of (a) the bird, (b) the fish, and (c) The Chinese knot. The conventional PAMPS gels and DN gels cannot form such complicated shapes. 60 The scale bars: $1 \mathrm{~cm}$.

(5mm in thickness and $9 \mathrm{~mm}$ in diameter) gels; the compression rate is $10 \% / \mathrm{min}$. The fracture energy $G$ was measured by tearing test on trousers-shaped samples standardized as the JIS-K6252 $1 / 2$ size (5mm in thickness, ${ }_{65} 50 \mathrm{~mm}$ in length (with $20 \mathrm{~mm}$ initial legs) and $7.5 \mathrm{~mm}$ in width) The tearing velocity was $250 \mathrm{~mm} / \mathrm{min}$. $G$, defined as the energy required for creating a unit area of fracture surface in a sample, is calculated by $G=F_{\text {ave }} / w$, where $F_{\text {ave }}$ is the average tearing resistance force, and $w$ is the width of the gel ${ }^{11}$. The 70 loading curves were measured by tensile test on dumbbellshaped gels standardized as the JIS-K6251-7 size (length: 
(a) PVA-DN gel
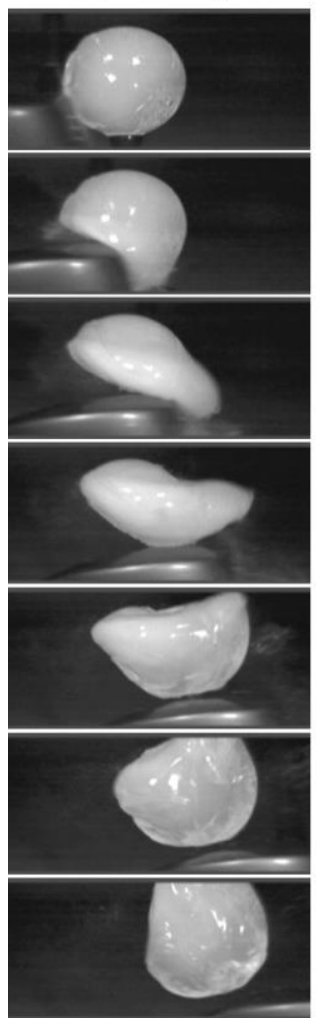

(b) PVA-PAMPS gel

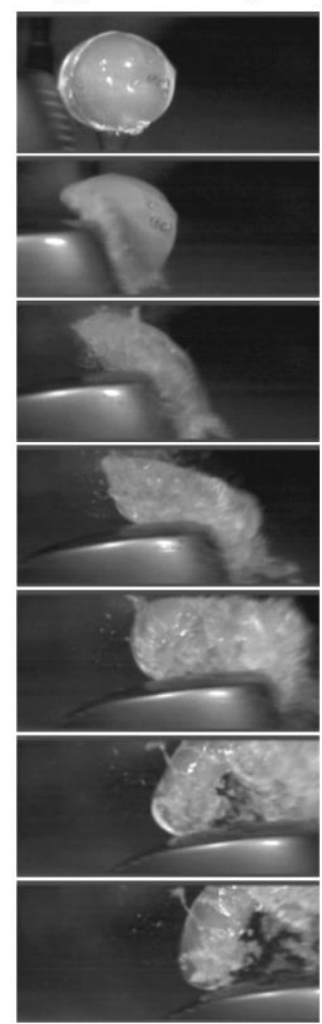

Fig. 2 High-speed camera images of the spherical (a) PVA-DN gel and (b) PVA-PAMPS gel hit by the golf club. Time interval between each picture is $3 / 4000 \mathrm{sec}$.

40mm; width: 10mm; thickness; 1.7-2.6mm; gauge length: $512 \mathrm{~mm}$; inner width; $2 \mathrm{~mm}$ ). The tensile velocity was $100 \mathrm{~mm} / \mathrm{min}$.

\section{Results and Discussion}

Fig. 1 shows the pictures of PVA-DN gels with various shapes. By this method, any complicated shapes can be 10 formed, such as the bird, the fish, and the Chinese knot shape depending on the shape of the PVA gels as the internal mold. In contrast, conventional PAMPS gels (and DN gels) cannot form even the simple ball-like shape. PAMPS gels were so fragile that they crashed whenever they were removed from 15 the external molds. Even though the PVA-DN gels have obtained the formability, their strength and toughness were still extremely high. Fig. 2 shows the pictures of the sphereshaped PVA-DN gel hit by a golf club (wood), recorded by a high-speed camera. In spite of the strong impact and the large 20 deformation, the PVA-DN gel recovered its original shape without breaking. On the contrary, the PVA-PAMPS gel was catastrophically broken by the impact. The full movies are available as the supporting informations. It is noted that the PVA-DN gels look a little bit opaque in contrast with 25 completely transparent common DN gels. This decrease of transparency is derived from the crystalline domain of PVA gels.

Here, we express the toughness of the PVA-DN gels by numeral values. Fig. 3 (filled bars) shows the fracture energy ${ }_{30} G$ of the PVA-DN, conventional DN, and PVA/PAAm DN

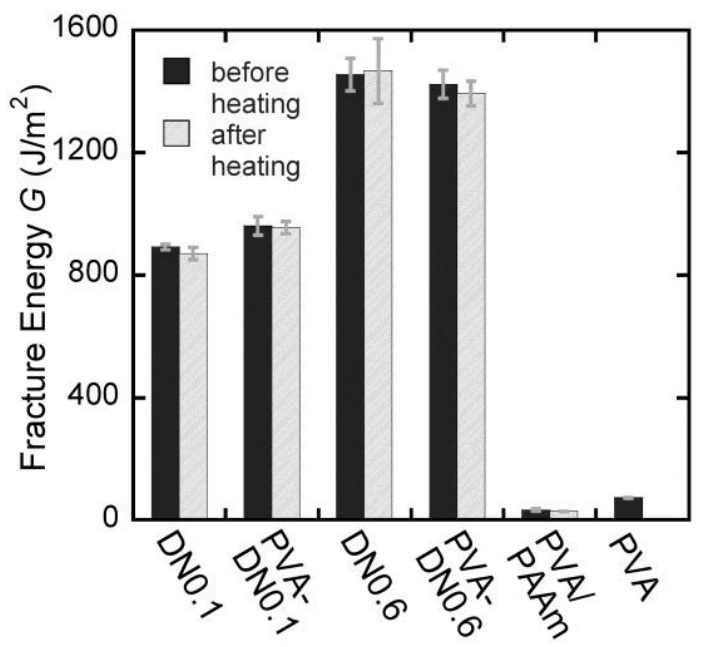

Fig. 3 The fracture energy $G$ of the PVA-DN gels, the conventional DN gels, the PVA-PAAm gel, and the PVA gel. The filled bars represent the $G$ of the DN gels before heating and the mesh bars represent the $G$ after heating. As the PVA gel was

35 dissolved by immersing in hot water, the $G$ of the PVA gel after heating could not be measured. The tearing velocity was $250 \mathrm{~mm} / \mathrm{min}$. PVA-DN denotes the PVA-DN gels, DN denotes the conventional DN gels, PVA/PAAm denotes the PVA/PAAm DN gels. The PAMPS gels and the PVA-PAMPS gels are too weak to

40 be measured.The following number denotes the initiator concentration for the PAMPS network (mol\%).

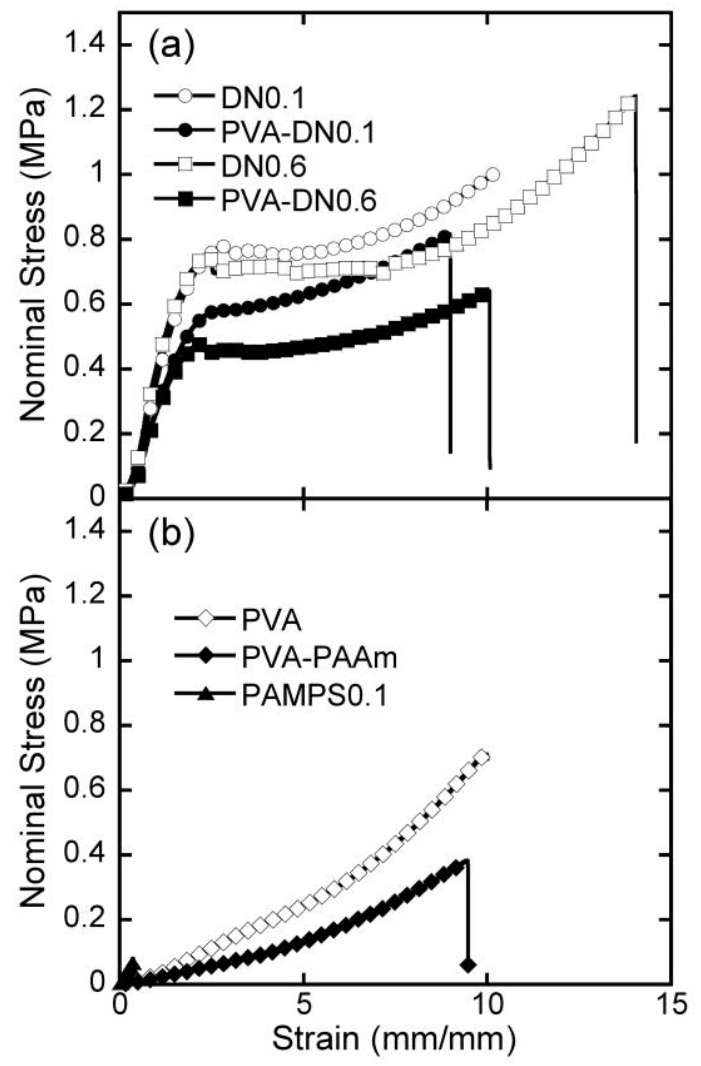

Fig. 4 The tensile stress-strain curves of (a) the PVA-DN gels, the conventional DN gels (b) the PVA-PAAm gel, the PVA gel, and the PAMPS gel measured by the tensile test. The PVA45 PAMPS gels are too weak to be measured. The tensile velocity was $100 \mathrm{~mm} / \mathrm{min}$. 
gels. The $G$, which represents the toughness, of the PVA-DN gels was anomalously high and similar to that of the conventional DN gels. On the other hand, even though the PVA/PAAm DN gels also show formability, their fracture 5 energy $G$ was quite lower than the other DN gels. Fig. 4 shows the tensile stress-strain curves of the DN gels. The tensile fracture stress of the PVA-DN gels was less than that of DN gels; on the other hand, the fracture strain of those gels was similar to each other. This phenomenon might be caused 10 by a lower PAMPS concentration in the PVA-DN or PVAPAMPS gels than conventional PAMPS gels and DN gels. As the PVA gels contained $10 \mathrm{wt} \%$ of polymer, concentration of AMPS in the PVA-PAMPS gels did not reach to $1 \mathrm{M}$ in spite of the gel immersion in 1M AMPS solution. Using slightly 15 higher concentration of AMPS solution may be useful to overcome the effect of PVA chains.

Next, we try to clarify the effect of the PVA chains to the toughness of the PVA-DN gels. Physical PVA gels are crosslinked by the formation of hydrogen bonds ${ }^{18}$ and these bonds 20 were easily destroyed by applying heat. Thus, the effect of PVA network to the mechanical properties of PVA-DN gels can be removed by heating the samples. So we investigated the effect of PVA network by measuring the fracture energy $G$ of the PVA-DN gels before and after heating. The DN gels, 25 the PVA-DN gels, and the PVA gel were immersed in hot water at $70^{\circ} \mathrm{C}$ for $8 \mathrm{~h}$. Fig. 3 shows the fracture energy $G$ of the DN gels before and after heating. The $G$ of the conventional DN gels and the PVA-DN gels did not change by this operation. This result implies that PVA network of PVA-DN ${ }^{30}$ gels has no effect to the mechanical strength of DN gels. The PVA network works only as "internal mold".

In order to explain why PVA-DN gels have both formability and toughness, we discuss the mechanical properties of the first network gels. First, we investigated the 35 mechanical properties of as-prepared gels in order to discuss formability. Fig. 5 shows the tensile loading curves of the PVA gel, which consists of neutral polymer and show formability, and the as-prepared PAMPS gels and PVAPAMPS gels, consisting of polyelectrolyte. The PVA gel 40 showed high flexibility, indeed it did not break even if the strain reached to 10 . In addition, its tensile fracture stress is relatively high. Therefore, PVA gels are not broken when they were ejected from complex molds. In contrast, the conventional PAMPS gels showed the completely different 45 behavior, rigid and brittle. They were broken when the stress attained only $0.08 \mathrm{MPa}$; thus, they cannot be removed from the mold without being broken. These data imply that the flexibility is so important for the formability.

Second, we take notice of the mechanical properties of 50 swollen first network gels. Our first paper about DN gels reports experimentally that the rigidness of the swollen first network PAMPS is enormously important for the strength of the DN gels ${ }^{7}$. This point is also indicated theoretically by some other papers ${ }^{21-25}$. As the index parameter of rigidness, 55 we use modulus for simplification. Fig. 6 shows the compressive stress-strain curves and the modulus of the PVA/PAMPS gels, the conventional PAMPS gels and PVA gel in swollen state. The conventional PAMPS gels showed

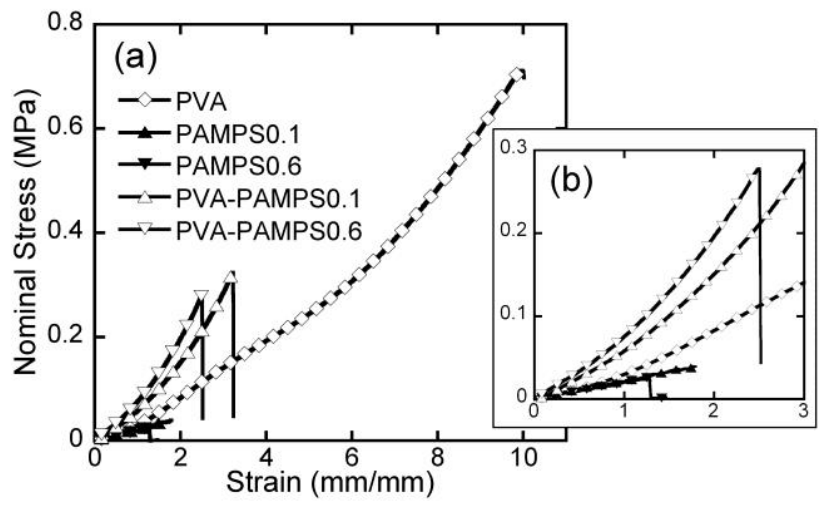

Fig. 5 (a) The tensile stress-strain curves of the PVA gel, the as60 prepared PAMPS gels, and the as-prepared PVA-PAMPS gels.

(b) The enlarged figure of the low-strain region.
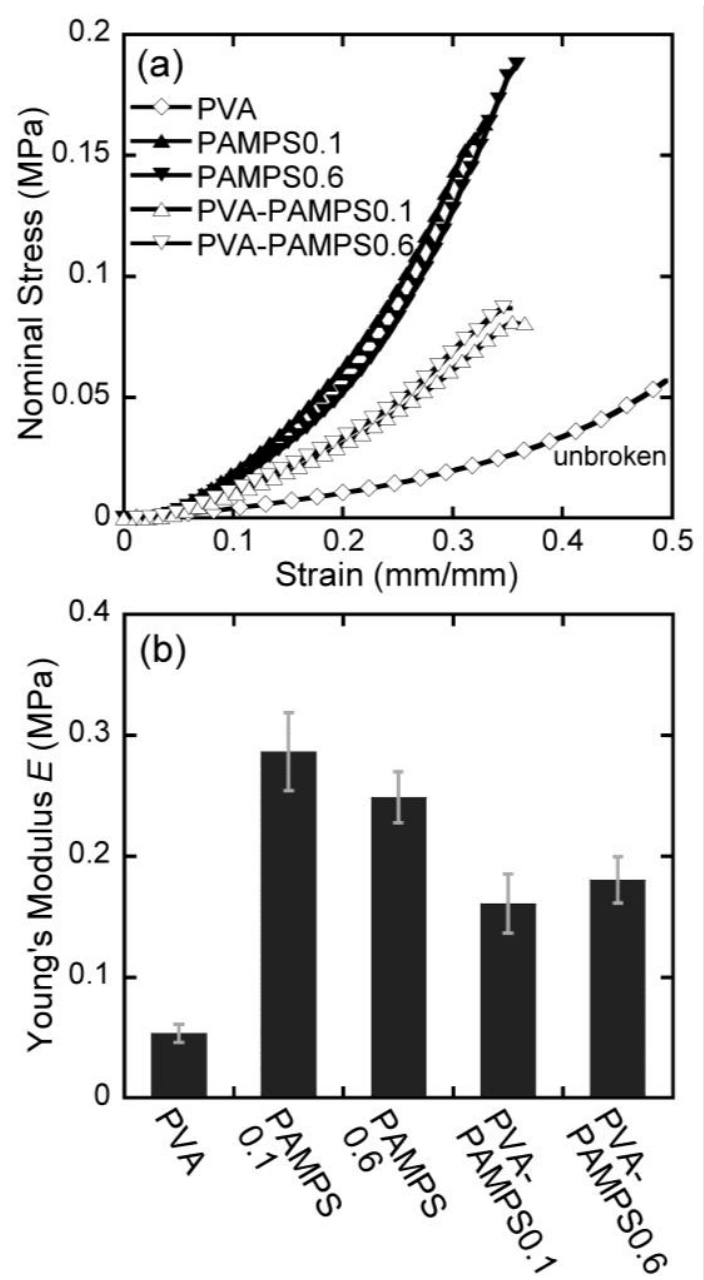

Fig. 6 (a) The compressive stress-strain curves and (b) the Young's modulus of the PVA gel, the swollen PAMPS gels, and the swollen PVA-PAMPS gels.

65 the highest moduli $(\sim 0.25 \mathrm{MPa})$. The PVA-PAMPS gels also showed the high moduli $(\sim 0.15 \mathrm{MPa})$, while the values were less than those of PAMPS gels. It is suggested that despite of the presence of PVA network, the properties of the swollen PVA/PAMPS gels are mainly dominated by rigid PAMPS 70 network, caused by fully stretching of polyelectrolyte chains. 
This rigidness leads the toughening of DN gels; thus, both the PVA-DN gels and the DN gels showed the toughness. In contrast, the modulus of the swollen PVA gel is quite low $(\sim 0.05 \mathrm{MPa})$ even though its polymer concentration is higher 5 than that of the swollen PAMPS gels. Thus, the PVA/PAAm DN gels do not show the toughness.

The essential points of this study are the following. Formability of (DN) gels is introduced by flexibility of asprepared first network gels. On the other hand, the extremely 10 high strength of DN gels is derived from the rigidness of swollen first network ${ }^{4}$. Until now, there has been no rigid and flexible gel in accordance with our best knowledge; thus, tough DN gels with formability have not been synthesized. For example, PAMPS/PAAm DN gels are strong but not 15 formable due to the rigid and "brittle" first network; on the other hand, PVA/PAAm DN gels show formability but weak due to the flexible and "soft" first network. This problem can be solved by using PVA/PAMPS gels as the first network. They are enough flexible (and soft) to form any shapes in as20 prepared PVA single network state; in contrast, after synthesizing PAMPS network and swelling, their property changes to rigid (and brittle), which enables to toughen (PVA)DN gels. This is the reason why PVA/PAMPS/PAAm (PVADN) gels have both formability and toughness.

\section{${ }_{25}$ Conclusions}

Free-shaped Double Network hydrogels (PVA-DN gels) were synthesized by using PVA gels as internal molds. They could form any complicated shapes, such as the fish and the bird. Additionally, the mechanical properties of PVA-DN gels 30 were almost the same as those of the conventional DN gels. These formability and the strength are provided via the difference of the mechanical properties between the flexible as-prepared PVA gels and the rigid swollen PVA/PAMPS gels. This study will enable DN gels to apply to biological and 35 industrial fields, such as artificial cartilages adopted into individual patient, artificial blood-vessels with complicated shapes, and nano-gel machines based on chemomechanical systems $^{26}$.

\section{Acknowledgments}

40 This work is supported by a Grant-in-Aid for the Specially Promoted Research (No. 18002002) from the Ministry of Education, Science, Sports and Culture of Japan.

\section{Notes and references}

1 T. Tanaka, Phys. Rev. Letters., 1978, 40, 820.

${ }_{45} 2$ J. P. Gong, M. Higa, Y. Iwasaki, Y. Katsuyama and Y. Osada, J. Phys. Chem. B, 1997, 101, 5487.; J. P. Gong, T. Kurokawa, T. Narita, G. Kagata, Y. Osada, G. Nishimura and M. Kinjo, J. Am. Chem. Soc., 2001, 123, 5582.

3 Y. Okumura and K. Ito, Adv. Mater., 2001, 13, 485.

${ }_{50} 4$ K. Haraguchi and T. Takeshita, Adv. Mater., 2002, 14, 1121.

5 J. P. Gong, Y. Katsuyama, T. Kurokawa and Y. Osada, Adv. Mater., 2003, 15, 1155.
6 M. Malkoch, R. Vestberg, N. Gupta, L. Mespouille, P. 55 Dubois, A. Mason, J. Hedrick, Q. Liao, C. Frank, K. Kingsbury and C. Hawker, Chem. Commun., 2006, 26, 2774.

7 T. Sakai, T. Matsunaga, Y. Yamamoto, C. Ito, R. Yoshida, S. Suzuki, N. Sasaki, M. Shibayama and U. Chung, Macromoleules, 2008, 41, 5379.

8 T. Nakajima, H. Furukawa, Y. Tanaka, T. Kurokawa, Y. Osada and J. P. Gong, Macromolecules, 2009, 42, 2184.

9 Y.-H. Na, T. Kurokawa, Y. Katsuyama, H. Tsukeshiba, J. P. Gong, Y. Osada, S. Okabe, T. Karino and M.

65 Shibayama, Macromolecules, 2004, 37, 5370.

10 A. J. Kerin, M. R. Wisnom and M. A. Adams, Proc. Inst. Mech. Eng. H, 1998, 212, 273.

11 Y. Tanaka, R. Kuwabara, Y.-H. Na, T. Kurokawa, J. P. Gong and Y. Osada, J. Phys. Chem. B, 2005, 109, 11559.

7012 H. Tsukeshiba, M. Huang, Y.-H. Na, T. Kurokawa, R. Kuwabara, Y. Tanaka, H. Furukawa, Y. Osada and J. P. Gong, J. Phys. Chem. B, 2005, 109, 16304.

13 M. Huang, H. Furukawa, Y. Tanaka, T. Nakajima, Y. Osada and J. P. Gong, Macromolecules, 2007, 40, 6658.

7514 C. Azuma, K. Yasuda, Y. Tanabe, H. Taniguro, F. Kanaya, A. Nakayama, Y. M. Chen, J. P. Gong and Y. Osada, J. Biomed. Mater. Res. A, 2007, 81, 373.

15 Y. Tanabe, K. Yasuda, C. Azuma, H. Taniguro, S. Onodera, A. Suzuki, Y. M. Chen, J. P. Gong.and Y.

80 Osada,. J. Mater. Sci.: Mater. Med., 2008, 19, 1379.

16 K. Yasuda, N. Kitamura, J. P. Gong, K. Arakaki, H. J. Kwon, S. Onodera, Y. M. Chen, T. Kurokawa, F. Kanaya, Y. Ohmiya and Y. Osada, Macromol. Biosci., 2009, 9, 307.

17 S.-H. Hyon, W.-I. Cha and Y. Ikada, Polymer Bulletin, $851989,22,119$.

18 N. A. Peppas and S. R. Stauffef, Journal of Controlled Release, 1991, 16, 305.

19 M. Ohta, A. Handa, H. Iwata, D. A. Rüfenacht and S. Tsutsumi, Technology and Health Care, 2004, 12, 225.

9020 T. Kurokawa, J. P. Gong and Y. Osada, Macromolecules, 2002, 35, 8161 .

21 Y.-H. Na, Y. Tanaka, Y. Kawauchi, H. Furukawa, T. Sumiyoshi, J. P. Gong and Y. Osada, Macromolecules, 2006, 39, 4641.

9522 R. Webber, C. Creton, H. R. Brown and J. P. Gong, Macromolecules, 2007, 40, 2917.

23 H. R. Brown, Macromolecules, 2007, 40, 3815.

24 Y. Tanaka, Europhys. Lett., 2007, 78, 56005.

25 Y. Tanaka, Y. Kawauchi, T. Kurokawa, H. Furukawa, T. 100 Okajima and J. P. Gong, Macromol. Rapid Commun., 2008, 29, 1514.

26 Y. Osada, H Okuzaki and H Hori, Nature, 1992, 355, 242.; M. Otake, Y. Kagami, M. Inaba and H. Inoue, Robot. Autonom. Syst., 2002, 40, 185.; R. Yoshida, T. Sakai, O.

105 Tabata and T. Yamaguchi, Sci. Tech. Adv. Mater., 2002, 3, 95.; M. E. Harmon, M. Tang and C. W. Frank, Polymer, 2003, 44, 4547 .

Address, Kita-10jo-Nishi-8chome, Kita-Ku, Sapporo, Hokkaido, Japan. 110 Fax: +81-11-706-2774; Tel: +81-11-706-2774; E-mail:

gong@mail.sci.hokudai.ac.jp 